\title{
RING AND LATTICE CHARACTERIZATIONS OF COMPLEX HILBERT SPACE
}

\author{
SHIZUO KAKUTANI ${ }^{1}$ AND GEORGE W. MACKEY
}

Introduction. In an earlier paper [1] $]^{2}$ by the authors it was suggested that at least the ring characterization of real Hilbert space given therein might be extended to the complex case by making use of a device employed by B. H. Arnold [2] in so extending a theorem of Eidelheit. It is the purpose of the present note to show that this can indeed be done not only for the ring characterization but for the lattice one as well.

The difficulty in the complex case is that the complex field admits a great many discontinuous automorphisms. It is overcome by making use of the device of Arnold mentioned above to show that in the infinite-dimensional case only continuous automorphisms present themselves (see Lemma 2 below). It is shown by an example that the infinite-dimensionality is essential and that accordingly the theorems of [1] cannot be extended to the complex case in quite their full generality.

1. Two preliminary lemmas. Lemmas 1 and 2 below constitute our formulation of Arnold's argument.

LemMa 1. Let $X$ be an infinite-dimensional normed linear space (real or complex). Then there exists an infinite sequence $x_{1}, x_{2}, x_{3}, \cdots$ of elements of $X$ such that given any bounded infinite sequence $\lambda_{1}, \lambda_{2}, \lambda_{3}, \cdots$ of scalars there exists a member $l$ of the conjugate space $\bar{X}$ of $X$ such that $l\left(x_{i}\right)=\lambda_{i}$ for $i=1,2, \cdots$.

Proof. As is well known, it is possible to construct infinite sequences $x_{1}, x_{2}, \ldots ; l_{1}, l_{2}, \ldots$ where each $x_{i}$ is in $X$ and each $l_{i}$ is in $\bar{X}$ so that $l_{i}\left(x_{j}\right)=\delta_{i}{ }^{i}$ for $i, j=1,2, \ldots$. Furthermore, it is clear that we may suppose that $\left\|l_{i}\right\|=1 / 2^{i}$. Let $\lambda_{1}, \lambda_{2}, \ldots$ be an arbitrary bounded sequence of scalars with l.u.b. $\left|\lambda_{i}\right|=M$. For each

Received by the editors January 28, 1946, and, in revised form, March 21, 1946.

1 It should be pointed out that Professor Kakutani is in no way responsible for the appearance of the results of this paper in their present form. They are the result of a continuation of our joint work based upon a suggestion which Professor Kakutani made in a letter just before he left for Japan and before the final draft of our earlier paper had been written. The second named author is entirely responsible for postponing the following up of this suggestion and publishing the results separately in the present paper.

2 Numbers in brackets refer to the bibliography. 
$x=l_{1}(x) x_{1}+l_{2}(x) x_{2}+\cdots+l_{n}(x) x_{n}$ in $x_{1}+x_{2}+\cdots$ define $l(x)$ as $l_{1}(x) \lambda_{1}+l_{2}(x) \lambda_{2}+\cdots+l_{n}(x) \lambda_{n}$. Then $l$ is obviously linear. ${ }^{8}$ Furthermore

$$
\begin{aligned}
|\bar{l}(x)| & \leqq\left\|l_{1}\right\|\|x\|\left|\lambda_{1}\right|+\left\|l_{2}\right\|\|x\|\left|\lambda_{2}\right|+\cdots+\left\|l_{n}\right\|\|x\|\left|\lambda_{n}\right| \\
& \leqq M\|x\|\left[1 / 2+1 / 4+\cdots+1 / 2^{n}\right]<M\|x\| .
\end{aligned}
$$

Thus $\bar{l}$ is bounded. Hence by the Hahn-Banach extension theorem or the Bohnenblust-Sobczyk extension of it to complex spaces [3], $\bar{l}$ coincides on $x_{1}+x_{2}+\cdots$ with a member $l$ of $\bar{X}$. Since it is obvious that $\bar{l}\left(x_{i}\right)=\lambda_{i}$ for $i=1,2, \ldots$ the truth of the lemma follows.

Lemma 2. Let $T$ be a one-to-one semi-linear ${ }^{4}$ transformation of an infinite-dimensional complex normed linear space $X$ onto a second such space $Y$. Then if $T$ carries closed maximal subspaces into closed maximal subspaces the automorphism involved in the semi-linearity of $T$ is a continuous one.

Proof. Let $x_{1}, x_{2}, \cdots$ be a sequence of elements of $X$ having the property described in Lemma 1 . Let $\mu_{1}, \mu_{2}, \ldots$ be an arbitrary bounded sequence of complex numbers and let $\mu_{1}^{\prime}, \mu_{2}^{\prime}, \ldots$ be their transforms by the automorphism in question. In order to prove that the automorphism is continuous it is clearly sufficient to show that $\left\{\mu_{i}^{\prime}\right\}$ is a bounded sequence. Suppose that it is not. Then we may select a subsequence $\lambda_{1}^{\prime}, \lambda_{2}^{\prime}, \ldots$ such that $\left|\lambda_{i}^{\prime}\right|>i\left\|T\left(x_{i}\right)\right\|$ for $i=1$, $2, \cdots$. Now let $l$ be a member of $\bar{X}$ such that $l\left(x_{i}\right)=\lambda_{i}$ for $i=1,2, \cdots$, let $M$ be the null space of $l$ and let $\bar{x}$ be an element of $X$ with $l(\bar{x})=1$. It is clear that for $i=1,2, \cdots$ we have $x_{i}=z_{i}+\lambda_{i} \bar{x}$ where $z_{i} \in M$. Hence $T\left(x_{i}\right) / \lambda_{i}^{\prime}=T\left(z_{i} / \lambda_{i}\right)+T(\bar{x})$. Since $\left\|T\left(x_{i}\right) / \lambda_{i}\right\|$ $<1 / i$ it follows that $T\left(-z_{i} / \lambda_{i}\right) \rightarrow T(\bar{x})$ as $i \rightarrow \infty$. Hence $T(\bar{x})$ is in the closure of $T(M)$. Since $M$ is a closed maximal subspace, $T(M)$ is, by hypothesis, closed. Hence $T(\bar{x}) \in T(M)$. Since $T$ is one-to-one this means that $\bar{x}$ is in $M$ and we have a contradiction. Thus the lemma is proved.

COROLLARY. Under the hypotheses of Lemma 2 the automorphism in question is either the identity or the automorphism carrying every complex number into its conjugate and hence $T$ is either linear or "conjugate linear."

2. The lattice characterization. Theorem 1 of [1] after slightly

3 In this paper linear means additive and homogeneous.

- A semi-linear transformation is a transformation $T$ such that $T(\lambda x+\mu y)=\lambda^{\prime} T(x)$ $+\mu^{\prime} T(y)$ where $x$ and $y$ are arbitrary elements of the vector space, $\lambda$ and $\mu$ are scalars and $\lambda \rightarrow \lambda^{\prime}$ is an automorphism of the scalar field independent of $x, y, \lambda$ and $\mu$. 
strengthening the dimensionality restriction may be stated for complex spaces as follows:

THEOREM 1. Let $X$ be an infinite-dimensional complex Banach space, and let $\Lambda$ be its lattice of closed subspaces. If there exists an operation $M \rightarrow M^{\prime}$ from $\Lambda$ to $\Lambda$ such that:

(1) if $M_{1}$ and $M_{2}$ are in $\Lambda$ and $M_{1} \subseteq M_{2}$ then $M_{1}^{\prime} \supseteq M_{2}^{\prime}$,

(2) if $M \in \Lambda$ then $M^{\prime \prime}=M$,

(3) if $M \in \Lambda$ then $M^{\prime} \cap M=0$,

then there exists an isomorphism between $X$ and $a$ (not necessarily separable) complex Hilbert space such that the correspondence $M \leftrightarrow M^{\prime}$ is carried over into the correspondence between orthogonal complements in the Hilbert space. In other words it is possible to introduce an inner product $(x, y)$ in $X$ with the properties listed in the standard Hilbert space axioms such that the new norm $\|x\|$ in $X$ defined by the equation $\|x\|=(x, x)^{1 / 2}$ is equivalent to the given norm $\|x\|$ and furthermore such that for each $M$ in $\Lambda, M^{\prime}$ is the set of all $y$ in $X$ for which $(x, y)=0$ for all $x$ in $M$.

Proof. Let $M$ be a one-dimensional subspace of $X$. It is clear that $M$ is closed and it follows from the fact that $M \rightarrow M^{\prime}$ is a lattice antiisomorphism that $M^{\prime}$ is a maximal closed linear subspace of $X$. Then let $M^{0}$ be the one-dimensional subspace of $\bar{X}$, the conjugate of $X$, consisting of those members $l$ of $\bar{X}$ such that $l(m)=0$ for all $m$ in $M^{\prime}$. It is easy to see that the operation $M \rightarrow M^{0}$ thus defined sets up a oneto-one linear independence preserving correspondence between the one-dimensional subspaces of $X$ on the one hand and those of $\bar{X}$ on the other. Now in the real case Lemma $A$ of [4] allowed us to conclude the existence of a linear transformation of $X$ on $\bar{X}$ such that $T(M)=M^{0}$ for all one-dimensional subspaces $M$ of $X$. A slight modification of the proof of this lemma enables us to conclude the existence of a semi-linear such transformation in the present case. We need only replace our reference to the theorem that a collineation between two real projective planes can be represented analytically by a linear transformation by a reference to the more general theorem relating collineations between projective planes over a field $\Phi$ to semi-linear transformations.

Now every maximal closed subspace of $X$ is clearly of the form $M^{\prime}$ where $M$ is one-dimensional and it is readily verified that $T\left(M^{\prime}\right)$ is the set of all $l$ in $\bar{X}$ taking $M$ into zero and hence is maximal and closed. It follows then from Lemma 2 that $T$ is either linear or conjugate linear. We show now that the former alternative is impossible. Supposing $T$ to be linear let $x$ and $y$ be linearly independent and set 
$T(x)=f, T(y)=g$. Then for each complex number $\mu, T(x+\mu y)=f+\mu g$ and $(f+\mu g)(x+\mu y)=f(x)+\mu(g(x)+f(y))+\mu^{2} g(y)$. Since $M \cap M^{\prime}=0$, $f(x), g(y)$ and $(f+\mu g)(x+\mu y)$ are all different from zero. But since $g(y) \neq 0$ the equation $f(x)+\mu(g(x)+f(y))+\mu^{2} g(y)=0$ has a solution for $\mu$. Since $(f+\mu g)(x+\mu y) \neq 0$ this is a contradiction.

We now use the conjugate linear transformation $T$ to define an inner product in $X$. For each two elements $x$ and $y$ let $(x, y)=f(x)$ where $f=T(y)$. Clearly $(x, y)$ is linear in $x$ and conjugate linear in $y$. To show that $(x, y)$ is Hermitian symmetric (that is, $\left.(y, x)=(x, y)^{*}\right)^{5}$ we observe first that $(x, y)=0$ if and only if $(y, x)=0$. In fact $(x, y)=0$ if and only if $M_{x} \subseteq M_{y}^{\prime}$ where $M_{x}$ and $M_{y}$ are the one-dimensional subspaces of $X$ generated by $x$ and $y$ respectively. But by properties (1) and (2) of the operation $M \rightarrow M^{\prime}$ we have $M_{x} \subseteq M_{y}^{\prime}$ if and only if $M_{y} \subseteq M_{x}^{\prime}$, that is, if and only if $(y, x)=0$. Now let $x$ and $y$ be arbitrary elements of $X$ with $(x, y) \neq 0$. Then there exist complex numbers $\lambda$ and $\mu$ such that (a) $\lambda(x, x)+(x, y)=0$ and (b) $\mu(y, y)+(x, y)=0$. (Since $g(z)$ where $g=T(z)$ is never zero for $z \neq 0$ it follows that for $z \neq 0,(z, z) \neq 0$.) Equation (a) may be transformed into equation (c): $\lambda(x, x)^{*}+(y, x)^{*}=0$ by means of the following sequence of equations each of which is obviously equivalent to its predecessor: $\lambda(x, x)+(x, y)=0,\left(x, \lambda^{*} x\right)+(x, y)=0,\left(x, \lambda^{*} x+y\right)=0,\left(\lambda^{*} x+y, x\right)=0$, $\lambda^{*}(x, x)+(y, x)=0, \lambda(x, x)^{*}+(y, x)^{*}=0$. Similarly (b) may be transformed into (d) $: \mu(y, y)^{*}+(y, x)^{*}=0$. Comparing (c) and (d) on the one hand and (a) and (b) on the other we conclude that $\lambda(x, x)^{*}=\mu(y, y)^{*}$ and that $\lambda(x, x)=\mu(y, y)$. Thus $(x, x) /(x, x)^{*}$ $=(y, y) /(y, y)^{*}$. It follows that if $(z, z)$ is real for one element $z$ it is real for every $w$ with $(x, w) \neq 0$ and hence for every $w$. But a simple adjustment of $T$ always enables us to realize this condition. Hence we may suppose that $(x, x)^{*}=(x, x)$ and $(y, y)^{*}=(y, y)$. We now conclude from (a) and (c) that $(y, x)^{*}=(x, y)$ so that $(x, y)$ is Hermitian symmetric. The proof of the positive definiteness of $(x, x)$ (after a suitable adjustment of $T$ ) and the fact that the norm which it defines has the properties required is word for word the same as the corresponding proof in the real case [1, page 52] and will not be repeated here. We only remark that it is readily verified, using the Bohnenblust-Sobczyk extension of the Hahn-Banach theorem, that the theorems of Banach referred to are true for complex as well as real spaces.

Just as in the real case we conclude at once, from Theorem 1 and certain obvious properties of finite-dimensional spaces, the truth of :

${ }^{5}$ In this paper whenever $A$ denotes a complex number $A^{*}$ will denote its complex conjugate. 
ThEOREM 2. Let $X$ be a complex Banach space and let $\Lambda$ be its lattice of closed subspaces. Then $X$ is isomorphic to a complex Hilbert space (not necessarily separable or infinite-dimensional) if and only if there exists an operation $M \rightarrow M^{\prime}$ from $\Lambda$ onto $\Lambda$ which satisfies (1), (2), and (3) of Theorem 1.

3. The ring characterization. The device for reducing the ring theorem to the lattice theorem used in the real case works equally well here and we may state the following theorem.

THEOREM 3. ${ }^{6}$ Let $X$ be a complex Banach space and let $R$ be the ring of continuous linear transformations of $X$ into itself. Then $X$ is isomorphic to a complex Hilbert space (not necessarily separable or infinitedimensional) if and only if there is an operation $T \rightarrow T^{\prime}$ from $R$ onto $R$ which has the following properties:

(1) If $T_{1}$ and $T_{2}$ are members of $R$ then

$$
\left(T_{1} T_{2}\right)^{\prime}=T_{2}^{\prime} T_{1}^{\prime} \text { and }\left(T_{1}+T_{2}\right)^{\prime}=T_{1}^{\prime}+T_{2}^{\prime} .
$$

(2) If $T$ is a member of $R$ then $T^{\prime \prime}=T$.

(3) If $T$ is a member of $R$ such that $T^{\prime} T=0$ then $T=0$.

In case $X$ is infinite-dimensional the analogue for rings of Theorem 1 is true. In fact if we follow through the construction of the lattice operation from the ring operation used in proving Theorem 3 from Theorem 2 (see [1, pp. 53 and 54]) and the construction of an inner product from the lattice operation we see without great difficulty that if $S_{x}$ and $S_{y}$ are members of $R$ whose ranges are the one-dimensional subspaces of $X$ defined by the elements $x$ and $y$ of $X$ then $(T(x), y)=0$ if and only if $S_{y}^{\prime} T S_{x}=0$ and $\left(x, T^{\prime}(y)\right)=0$ if and only if $S_{x}^{\prime} T^{\prime} S_{y}=0$. Thus since $\left(S_{y}^{\prime} T S_{x}\right)^{\prime}=S_{x}^{\prime} T^{\prime} S_{y}$ it follows that $(T(x), y)$ $=0$ if and only if $\left(x, T^{\prime}(y)\right)=0$ and hence that $\left(x, T^{\prime}(y)\right)=0$ if and only if $\left(x, T^{*}(y)\right)=0$ where $T^{*}$ is the adjoint of $T$ calculated with respect to the inner product $(x, y)$. Thus for every $y, T^{\prime}(y)$ and $T^{*}(y)$ have identical orthogonal complements so that one is a scalar multiple of the other. It follows easily from this that $T^{\prime}=T^{*}$, for all $T$ in $R$. In fact for each $x$ in $X,(1+T)^{\prime}(x)=\lambda(1+T)^{*}(x)=\lambda\left(1+T^{*}\right)(x)$ $=\lambda x+\lambda T^{*}(x)$ and $(1+T)^{\prime}(x)=\left(1+T^{\prime}\right)(x)=x+\mu T^{*}(x)$. Thus $\lambda x+\lambda T^{*}(x)=x+\mu T^{*}(x)$ and if $T^{*}(x)$ and $x$ are linearly independent we can conclude that $\lambda=\mu=1$. If $T^{*}(x)$ and $x$ are linearly dependent choose $S$ so that $S^{*}(x)$ and $x$ are not. Then $(S+T)^{*}(x)=(S+T)^{\prime}(x)$ so that $S^{*}(x)+T^{*}(x)=S^{\prime}(x)+T^{\prime}(x)$. Also $S^{*}(x)=S^{\prime}(x)$ so that

${ }^{6}$ For a proof of a weaker form of this theorem in which it is assumed in addition that $(\lambda T)^{*}=\lambda^{*} T^{*}$ see $[5]$. 
$T^{*}(x)=T^{\prime}(x)$. Thus in any case $T^{*}(x)=T^{\prime}(x)$ so that $T^{*}=T^{\prime}$. Thus we may state:

Theorem 4. Let $X$ be an infinite-dimensional complex Banach space and let $R$ be the ring of all continuous linear transformations of $X$ into itself. If there exists an operation $T \rightarrow T^{\prime}$ from $R$ onto $R$ which has properties (1), (2) and (3) of Theorem 3 so that $X$ is isomorphic to a not necessarily separable complex Hilbert space then this isomorphism may be set up in such a way that the correspondence $T \rightarrow T^{\prime}$ goes over into the correspondence between an operator and its adjoint. In other words, it is possible to introduce an inner product $(x, y)$ in $X$ with the properties listed in the standard Hilbert space axioms such that the new norm \|\|$x \mid \|$ in $X$ defined by the equation $\|x \mid\|=(x, x)^{1 / 2}$ is equivalent to the given norm $\|x\|$ and furthermore such that for all $x$ and $y$ in $X$, $(T(x), y)=\left(x, T^{\prime}(y)\right)$.

4. Some counter examples. The discussion given in paragraph 3 of [1] applies without essential change in the complex case so that we may conclude here as there that our theorems need not be true if we fail to assume completeness for $X$.

Finally we show that the infinite-dimensionality assumption in Theorems 1 and 4 cannot be omitted. To this end let $\alpha$ be any automorphism of the complex field other than the identity and the conjugacy automorphism. For each complex number $\lambda$ let $\lambda^{\prime}=\alpha^{-1}(\alpha(\lambda))^{*}$. Then $\lambda \rightarrow \lambda^{\prime}$ is clearly an involutory automorphism of the complex field. Furthermore if $\lambda_{1} \lambda_{1}^{\prime}+\lambda_{2} \lambda_{2}^{\prime}+\cdots+\lambda_{n} \lambda_{n}^{\prime}=0$ then

$$
\alpha\left(\lambda_{1}\right) \alpha\left(\lambda_{1}^{\prime}\right)+\alpha\left(\lambda_{2}\right) \alpha\left(\lambda_{2}^{\prime}\right)+\cdots+\alpha\left(\lambda_{n}\right) \alpha\left(\lambda_{n}^{\prime}\right)=0
$$

or

$$
\alpha\left(\lambda_{1}\right) \alpha\left(\lambda_{1}\right)^{*}+\alpha\left(\lambda_{2}\right) \alpha\left(\lambda_{2}\right)^{*}+\cdots+\alpha\left(\lambda_{n}\right) \alpha\left(\lambda_{n}\right)^{*}=0 .
$$

Hence $\alpha\left(\lambda_{1}\right)=\alpha\left(\lambda_{2}\right)=\cdots=\alpha\left(\lambda_{n}\right)=0$ so that $\lambda_{1}=\lambda_{2}=\cdots=\lambda_{n}=0$. Now let $R_{n}$ be the vector space of $n$-uples of complex numbers and for each two members $x=\lambda_{1}, \lambda_{2}, \cdots, \lambda_{n} ; y=\mu_{1}, \mu_{2}, \cdots, \mu_{n}$ of $R_{n}$ let $(x, y)=\lambda_{1} \mu_{1}^{\prime}+\lambda_{2} \mu_{2}^{\prime}+\cdots+\lambda_{n} \mu_{n}^{\prime}$. Then $(x, y)$ is linear in $x$, semilinear with respect to ' in $y$ and such that $(y, x)=(x, y)^{\prime}$ and $(x, x)=0$ if and only if $x=0$. Now for each linear transformation $T$ of $R_{n}$ into itself define $T^{\prime}$ by the equation $(T(x), y)=\left(x, T^{\prime}(y)\right)$ for all $x$ and $y$ in $R_{n}$. Then, as is easily seen, $T^{\prime}$ is a linear transformation of $R_{n}$ into itself and the operation $T \rightarrow T^{\prime}$ has the properties (1), (2), and (3) listed in the statement of Theorem 3. On the other hand it is not difficult to show that there is no inner product in $X$ for which $T^{\prime}=T^{*}$. Let $\lambda$ be a non-real complex number such that $\alpha(\lambda)$ is real. Then 
$\lambda^{\prime}=\lambda$. Hence $\lambda \rightarrow \lambda^{\prime}$ is not the conjugacy operation. Since it is obviously not the identity there exists a non-real complex number $\mu$ such that $\mu^{\prime}$ is real. Let $T$ be $\mu$ times the identity. Then $T^{\prime}$ will be $\mu^{\prime}$ times the identity and for any inner product $T^{*}$ will be $\mu$ times the identity. Since $\mu^{\prime}$ is real and $\mu$ is not, $T^{\prime}$ and $T^{*}$ cannot be equal.

Similarly using this same "semi-inner product" to define orthogonality in $R_{n}$ one can prove for $n>1$ the existence of an operation on subspaces having the properties listed in the statement of Theorem 1 and not coinciding with the operation of taking orthogonal complements for any inner product in $R_{n}$.

\section{BIBLIOGRAPHY}

1. S. Kakutani and G. W. Mackey, Two characterizations of real Hilbert space, Ann. of Math. vol. 45 (1944) pp. 50-58.

2. B. H. Arnold, Rings of operators on vector spaces, Ann. of Math. vol. 45 (1944) pp. 24-49.

3. H. F. Bohnenblust and A. Sobczyk, Extensions of functionals on complex linear spaces, Bull. Amer. Math. Soc. vol. 44 (1938) pp. 91-93.

4. G. W. Mackey, Isomorphisms of normed linear spaces, Ann. of Math. vol. 43 (1942) pp. 244-260.

5. Y. Kawada, Über den Operatorenring Banachscher Raume, Proc. Imp. Acad. Tokyo vol. 19 (1943) pp. 616-621.

Mathematical INSTITUTe, Osaka Imperial UNIVERSITY AND HARVARD UNIVERSITY 